\title{
Snowballing Technique for High Flow Arteriovenous Fistula: A Technical Note
}

\author{
Riyadh Nasser Alokaili, MBBS, ABR, CAQ, $\mathrm{PhD}^{1}$, Hesham Riyadh Alokaili, $\mathrm{MBBS}^{2}$, \\ Mohammad F. Badran, MBBS, FRCR, FFR, FRCSI ${ }^{1}$, Homoud Abdulaziz Aldahash, MBBS 3 , \\ Shagran M. Binkhamis, MBBS \\ ${ }^{1}$ Department of Radiology, King Faisal Specialist Hospital and Research Center, Riyadh, Saudi Arabia \\ ${ }^{2}$ National Guard Health Affairs Hospital, Riyadh, Saudi Arabia \\ ${ }^{3}$ Department of Neurosciences, King Faisal Specialist Hospital and Research Center, Riyadh, Saudi Arabia
}

A novel endovascular technique to occlude high flow direct arteriovenous fistulae is presented, where the distal tip of the microcatheter acts as a nucleus that the operator can grow a plug from a liquid embolic agent. Its advantages (such as cost-saving and distal reachability), disadvantages (such as embolic material instability), and technique are discussed.

Key Words: Endovascular technique; Arteriovenous fistula; Interventional radiology

\section{Correspondence to:} Hesham Riyadh Alokaili, MBBS National Guard Health Affairs Hospital, MBC 28, PO Box 3354, Riyadh 11211, Saudi Arabia Tel: +966507240772

Fax: +96614414839

E-mail: Hishamalokaili@gmail.com

Received: June 5, 2021

Revised: January 14, 2022

Accepted: January 23, 2022

Copyright $\odot 2022$ Korean Society of Interventional Neuroradiology This is an Open Access article distributed under the terms of the Creative Commons Attribution Non-Commercial License (http://creativecommons.org/licenses/by-nc/4.0) which permits unrestricted non-commercial use, distribution, and reproduction in any medium, provided the original work is properly cited.

pISSN 2093-9043 eISSN 2233-6273 
at a curve. Lowering the blood pressure is encouraged at this point. At this stage, a small amount of liquid embolic, prefer- ably of the non-adhesive variety, is injected. The objective of the small volume is to be sufficiently small that the force that

\section{Table 1. Summary of recommendations before and instruction steps during performance of the snowballing embolization technique}

Preparatory recommendations prior to snowballing

1. We advise against this technique if imaging equipment is of poor quality

2. Anesthetizing and paralyzing the subject is advised

3. Lowering the blood pressure is encouraged (it was not manipulated in this particular case)

4. If the tip of the catheter/microcatheter flutters or pulsates, consider repositioning it to a less turbulent location to limit its motion
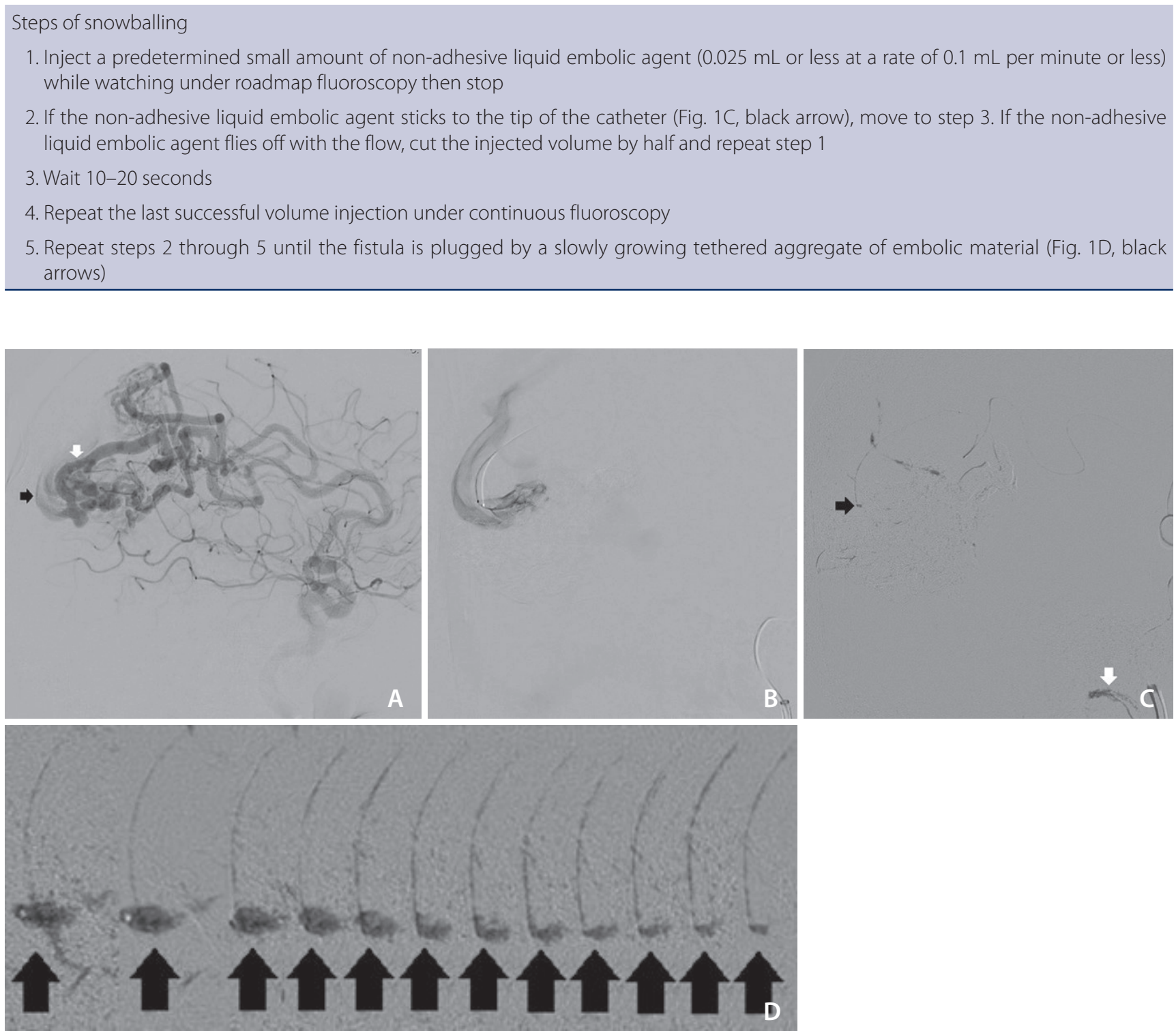

Fig. 1. Describing radiography obtained during a 'snowballing procedure' for arteriovenous fistula. (A) Lateral view of the right internal carotid angiogram. A direct fistula connects a hypertrophied feeding artery (white arrow) to a hypertrophied draining vein (black arrow). (B) Pre-embolization lateral view of a super-selective left anterior cerebral artery feeder angiographic run illustrating a direct fistula. (C) Lateral fluoroscopic roadmap image of initial embolic agent aggregate hanging on the tip of the microcatheter (black arrow). Embolic agent in the jugular bulb from prior standard technique (white arrow). (D) Intermittent lateral subtracted fluoroscopic images with 20-second separation between each spot image with a right to left time course of earlier to later, showing incremental growth of the embolic agent snowball at the tip of the microcatheter (black arrows). 
keeps it attached to the microcatheter is not exceeded by the drag force of the flowing blood. The 10-20 second wait is to allow the embolic agent time to solidify as it sets, and by that increase its force of attachment to the microcatheter (in the case of ONYX, by losing its dimethyl sulfoxide solvent in the blood stream, leaving behind the solidified/precipitated co-polymer). These steps are repeated to build an ever-enlarging catheter anchored embolic mass until the inflow is plugged, as summarized in Table 1.

Consider an alternative technique if the tethered embolic material aggregate detaches prematurely after 1 or more attempts, depending on context and the operator's best judgment. The authors intuitively without evidence feel that after 3 attempts the odds of success are slim.

\section{CASE PEPORT}

A patient presented to our center for treatment of a large (Spetzler-Martin grade IV-V) left occipital-parietal arteriovenous malformation with a nidus that harbors direct arteriovenous high flow shunts. The largest of these fistulae was from a hypertrophied left anterior cerebral artery feeder measuring approximately $5 \mathrm{~mm}$ in diameter (Fig. 1A). A microcatheter (Apollo 1.5 French 5-centimeter detachable tip [eV3, Irvine, CA, USA]) was distally navigated to this feeder (Fig. 1B). The initial injection of a non-adhesive liquid embolic agent (ONYX-18 [eV3]) was noticed to fly through the fistula unimpeded with some droplets reaching the jugular bulb (Fig. 1C, white arrow). So, the standard injection technique (injections with visual stop cues) was abandoned in favor of the snowballing technique (injections with predetermined volume stop cues).

\section{DISCUSSION}

How the snowball grows is largely speculative. We presume that the non-adhesive liquid embolic agent at the distal tip of the microcatheter solidifies as it is exposed to blood and that the tip of the catheter offers a suitable surface where the non-adhesive liquid embolic agent can initially implant on. Then we presume that with each small incremental agent injection a new layer is added to the one before. Without a way for us to know for certain, it can be hypothesized that the new layer is added inside the injection before to cause it to grow (similar to how a multi-layer skin and mucosal layers are arranged, older is superficial and newer is deep), or and more likely, the new layer goes on top or outside the preceding layer (similar to how a snowball grows, older is central and newer is peripheral).

When the aggregate is small the dominant force acting on its molding is the blood flow. Notice that the initial right 7 images of the aggregated embolic agent in Fig. 1D are pointing anteriorly and elongated, which happens to be the same force vector of blood flow. When the aggregate reaches a critical mass, gravity becomes a more important molding force, which accounts for the increased amount of embolic substance posterior to the microcatheter on the last left 5 images of Fig. 1D.

This technique is not immune to criticisms. One such critic is the risk of premature detachment of a large aggregate that might be more harmful to the lung compared to the small embolic droplets used in the standard technique. Another disadvantage is the inherent instability of the snowball until a plug is achieved. There is also the additional time and radiation needed to execute this task. On the other hand, snowballing can be cost-saving as it may not necessitate using a balloon (e.g., balloon-assisted embolization) or placing large expensive embolic devices such as plugs or coils (e.g., pressure cooker technique) to slow down the flow. ${ }^{2,3}$ It also might be the preferable option for fistulae that are only reachable with floppy microcatheters that cannot deliver non-liquid embolic options, and, therefore, alternative methods such as balloon-assisted embolization and the pressure cooker technique are not possible., ${ }^{2,3}$ Additionally, this proposed predetermined volume stop cue technique can be used under a poor image quality environment when visual stop cue techniques are not suitable.

Hypothetically, the technique might be made more effective by using a larger diameter catheter as bigger objects offer a larger surface to implant the aggregate on. Also, greater viscosity embolic material should be less vulnerable to flow forces. Combining the snowball technique with other known techniques (e.g., blood pressure reduction or mechanical compression when feasible) is likely to have additive value.

The technique was used 3 times by the authors with no complications. Technical success was achieved in 2 and in 1 attempt it was abandoned for an alternative technique. 


\section{CONCLUSION}

In conclusion, this technique may be used under poor image quality when visual stop cues are unfavorable. It may also be preferable in situations where non-liquid embolic agents cannot be delivered such as with the usage of floppy micro-catheters. This technique also has cost-saving benefits when compared to the usage of plugs or coils. A drawback to this technique is a higher potential harm from premature dislodgement due to the size of the embolic agent aggregate. In addition, execution of this technique requires more time and radiation. All in all, we believe this technique to have utility in the treatment of high-flow arteriovenous fistulas.

\section{Fund}

None.

\section{Ethics Statement}

Our local review board equivalent (Review Advisory council) approve this paper. Since the consent for publication was not available for the patients mentioned in the figures and the main text, patients' information was anonymized by removing the sex and specific age. Our institution IRB waives approval for case reports with less than 2 cases.

\section{Conflicts of Interest}

The authors have no conflicts to disclose.

\section{Author Contributions}

Concept and design: RNA, HRA, and SMB. Analysis and interpretation: RNA, HRA, MFB, HAA, and SMB. Data collection: RNA, HRA, and HAA. Writing the article: RNA, HRA, and MFB. Critical revision of the article: RNA, HRA, MFB, HAA, and SMB. Final approval of the article: RNA, HRA, MFB, HAA, and SMB. Overall responsibility: RNA, HRA, MFB, HAA, and SMB.

\section{ORCID}

Riyadh Nasser Alokaili: https://orcid.org/0000-0001-7922-0812 Hesham Riyadh Alokaili: https://orcid.org/0000-0001-9333-1922 Mohammad F. Badran: https://orcid.org/0000-0003-3664-5284 Homoud Abdulaziz Aldahash: https://orcid.org/0000-00026186-0747

Shagran M. Binkhamis: https://orcid.org/0000-0001-7521-3084

\section{REFERENCES}

1. Herman TE, Siegel MJ, Vachharajani A, Masand P, Cross D. Cerebral arteriovenous fistula to pulmonary artery onyx embolization. J Perinato/ 2007;27:238-240

2. Jagadeesan $B D$, Grigoryan $M$, Hassan $A E$, Grande AW, Tummala RP. Endovascular balloon-assisted embolization of intracranial and cervical arteriovenous malformations using dual-lumen coaxial balloon microcatheters and Onyx. Oper Neurosurg 2013;73:238-243

3. Chapot R, Stracke P, Velasco A, Nordmeyer H, Heddier M, Stauder $\mathrm{M}$, et al. The pressure cooker technique for the treatment of brain AVMs. J Neuroradiol 2014;41:87-91 